\title{
Quantitative Comparison of Two Cortical Surface Extraction Methods Using MRI Phantoms
}

\author{
Simon F. Eskildsen and Lasse R. Østergaard \\ Dept. of Health Science and Technology, Aalborg University, Denmark
}

\begin{abstract}
In the last decade several methods for extracting the human cerebral cortex from magnetic resonance images have been proposed. Studies comparing these methods have been few. In this study we compare a recent cortical extraction method with FreeSurfer, which has been widespread in the scientific community during recent years. The comparison is performed using realistic phantoms generated from surfaces extracted from original brain scans. The geometrical accuracy of the reconstructed surfaces is compared to the surfaces extracted from the original scan. We found that our method is comparable with FreeSurfer in terms of accuracy, and in some cases it performs better. In terms of speed our method is more than 25 times faster.
\end{abstract}

\section{Introduction}

Reconstruction of the human cerebral cortex from magnetic resonance (MR) images facilitates morphometric studies and brain mapping, and provides intuitive visualisation of the human brain for the use in e.g. surgical planning. Since the nineties a number of algorithms has been developed for extracting the boundaries of the cortex from MR images [12345677. FreeSurfer has been around for more than seven years, and has, due to the fact that it is freely available, become widespread in the scientific community. We have recently published a method (henceforth designated Fast Accurate Cortex Extraction (FACE)), which resembles FreeSurfer in many aspects, but is significantly improved in terms of computational speed 89].

When performing morphometric studies the accuracy of the cortex reconstructions is very important. Therefore, it is of interest to investigate how well ACE performs in terms of accuracy compared to FreeSurfer. Quantification of the accuracy is difficult as the ground truth is rarely available. A means to measure the accuracy is using phantoms resembling real neuroanatomical data. Lee et al. 10] compared FreeSurfer [4, CLASP [7] and BrainVISA 2] using generated phantoms. They found that CLASP was more accurate than BrainVISA and FreeSurfer. However, CLASP is not publicly available, while the two other methods are. FreeSurfer performed second best in the study. In this study we compare our method, ACE, to FreeSurfer using realistic phantoms generated from real MR scans. 


\section{Methods}

To evaluate the two cortex extraction methods, eight healthy young subjects (age: $32 \pm 7.4$ ) and eight healthy middle-aged subjects (age: $54.3 \pm 6.0$ ) were selected, and a comparison method similar to the method described by Lee et al. was used [10]. For each subject both methods were used to extract the cortical boundaries. The surfaces extracted by each method were used as reference for the generation of simulated MR scans as described below. The cortex of these customised phantoms were extracted by each method and the resulting surfaces were compared to the reference surfaces (see figure 1).

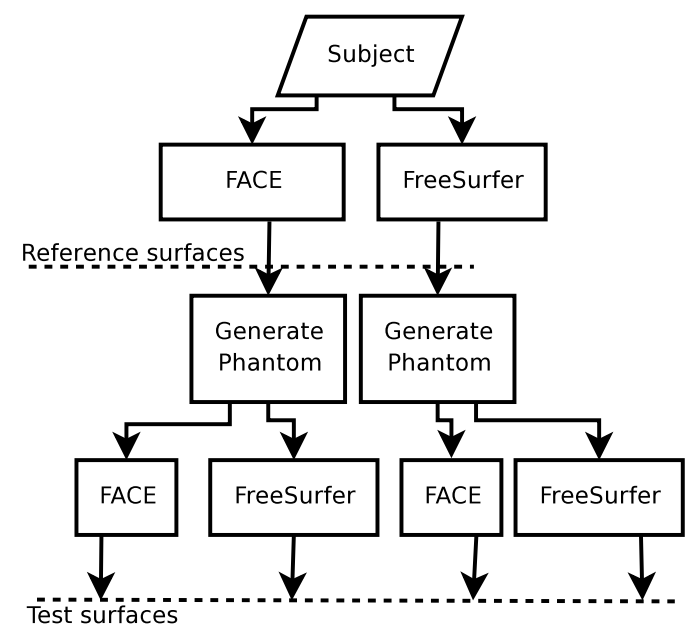

Fig. 1. Flow chart illustration of the comparison method

The following briefly describes the two cortex extraction methods, the generation of the test phantoms, and how the error between the reference surfaces and the test surfaces was quantified.

\subsection{FreeSurfer Method}

FreeSurfer 411] first registers the input MR volume to Talairach space 12. Nonuniformities originating from inhomogeneities in the magnetic field are corrected, and the intensities are normalised. The resulting volume is skull stripped using an approach similar to BET [13]. The WM voxels inside the skull stripped volume is labelled using a two-step segmentation algorithm based on intensities and prior knowledge of the GM/WM interface. The ventricles and subcortical matter inside the WM component is filled, and the WM is separated into the two hemispheres by a sagittal cut through the corpus callosum and an axial cut through the 
pons. A connected component algorithm is used to isolate the main body of WM voxels, i.e. the cerebrum WM voxels.

From the WM voxels a surface mesh is constructed by generating connected triangles on the faces of the voxels. The resulting surface for each hemisphere is topology corrected to be isomorph to a sphere, and a deformation process smoothes the surface while maintaining it at the WM/GM interface. The pial, or GM surface is found by displacing the WM surface toward the GM/CSF interface using the local surface normals and intensity gradients.

\subsection{Fast Accurate Cortex Extraction Method}

FACE performs similar preprocessing steps as FreeSurfer. The registered, intensity corrected, and skull stripped volume is segmented into WM, GM, and CSF using a fuzzy clustering algorithm solely based on the intensities, and a WM labelling is performed by maximum membership classification. Cerebellum and the brain stem is removed using atlas information, and the hemispheres are separated by a sagittal cut through the corpus callosum. After a connected component analysis spherical topology of each hemisphere is obtained using a topology correction algorithm [14, and the WM hemispheres can be tessellated by an iso-surface algorithm yielding surfaces with Euler characteristics of a sphere (genus=0).

The iso-surface generated from the WM cerebrum voxels are deformed to fit the WM/GM interface under the influence of smoothing forces and forces derived from the surface normals, the fuzzy voxel classification, and gradient information of the original image.

The GM surface is found using the method described in [9]. The WM surface is displaced towards the GM/CSF interface using a combination of the local surface normals and a gradient vector field calculated from an edge map of the voxel segmentation. The influence of the two vector force fields on each vertex in the surface is weighted by the curvature of the surface, which enables different deformation behaviour according the position on the surface (sulcus or gyrus). The deformation is not minimising an objective function, which means that the complexity is low compared to the deformation process in FreeSurfer.

\subsection{Phantom Generation}

Membership volumes of WM, GM, and CSF were generated directly from the extracted surfaces. This was accomplished by labelling each voxel completely inside the WM surface as WM, and calculating the inside fraction of each voxel intersected by the surface. This was also done for the GM surface, and the memberships for the three tissue classes were calculated from the fuzzy labelled volumes (see figure 2). The three membership volumes were used as input to an MRI simulator [15] with the same acquisition parameters as the original MR scans $(\mathrm{TR}=18 \mathrm{~ms}, \mathrm{TE}=10 \mathrm{~ms}, 1 \mathrm{~mm}$ slices $)$. The intensities of the resulting volume were normalised to the range of the original scan. Finally, subcortex, ventricles, cerebellum, brain stem, and extra-cerebral tissue were added from the original scan by superimposing the simulated brain scan onto the original (figure 3). 

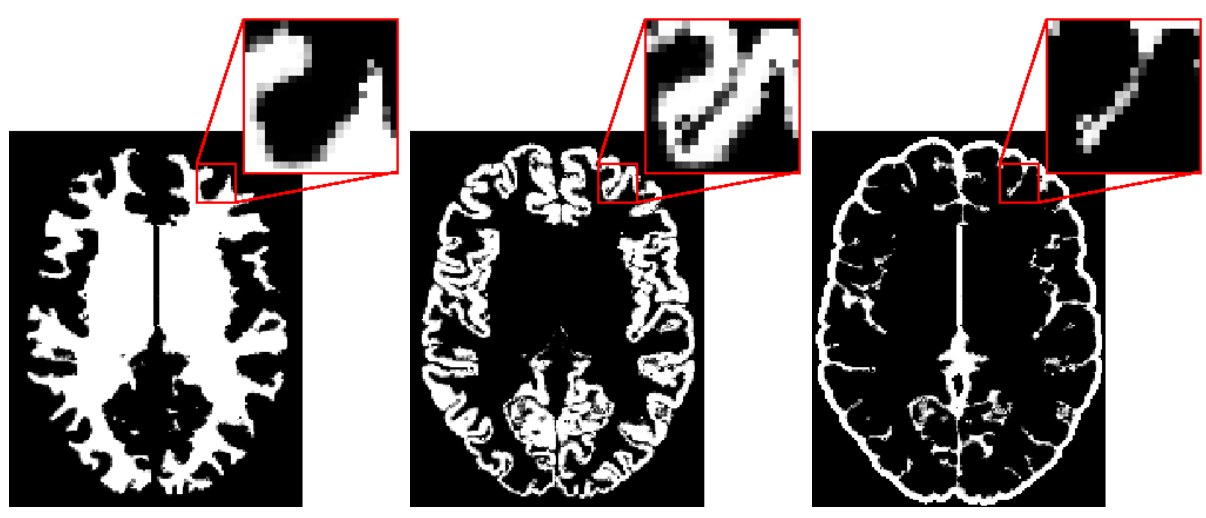

Fig. 2. Fuzzy membership volumes generated from the extracted surfaces. Left to right: WM, GM, and CSF.
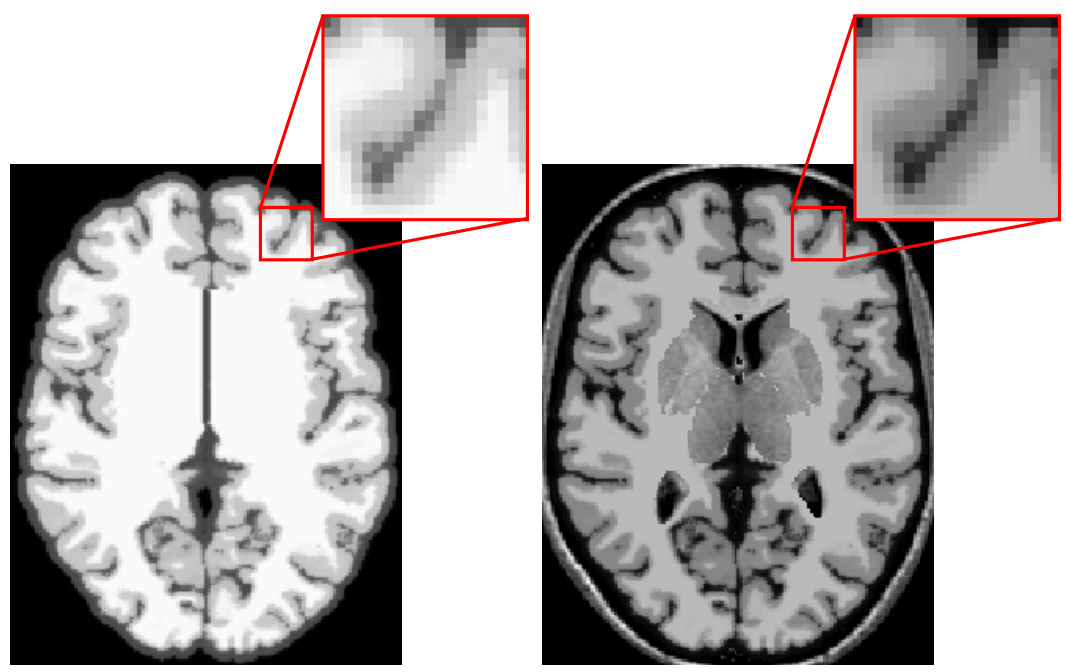

Fig. 3. Phantom produced by the MRI simulator (left), and final phantom after normalisation and added original tissue (right).

\subsection{Accuracy Assessment}

To test the accuracy of each method, reconstructions of the cortical boundaries were generated from the 32 phantoms. The reconstructions were then compared to the reconstructions of the original MR scans. Both methods ensures correct topology by volume- or surface-correction. Thus the comparison was based solely on geometrical factors. Four factors were considered, namely volume difference, surface area difference, over/under segmentation ratio, and the explicit geometrical error. Also the vertex density was taking into consideration in the comparison. 
- Volume Difference: The enclosing volume of the surfaces was calculated and the difference (in percent) from the reference surfaces was measured.

- Surface Area Difference: Surface areas were calculated and the difference (in percent) from the reference surfaces was measured.

- Over/under segmentation ratio: Tissue membership volumes of WM, GM and CSF were created from the test surfaces similar to the procedure used in the phantom generation. The resulting fuzzy maps were compared to the maps generated from the reference surfaces, and the percentages of voxels respectively missing inside (false negatives) and added outside (false positives) the reference map were calculated.

- Explicit Geometrical Error: The Euclidean distance from each vertex in the reference surface to the closest face on the test surface was measured. The root mean square error of these distances was calculated for both the WM surface and the GM surface. Similarly, the distance was measured from the test surface to the reference surface. The latter was done to avoid that simply adding vertices to the surface did not necessarily reduce the error.

\section{$3 \quad$ Results}

The cortical extractions were performed on an AMD Opteron 2.6 GHz processor with 12 GB memory. The average extraction time from native scan to final surfaces for FreeSurfer was 20.1 hours, while it was 0.8 hours for FACE. The following presents the results on how well the methods reconstructed the original surfaces from the generated phantoms.

When comparing the reconstructed surfaces visually, only small differences can be discerned. Figure 4 shows the original GM surface along with the reconstructions by the two methods. The number of vertices in the surfaces generated by the two methods vary. FreeSurfer generates surfaces with almost twice the number of vertices compared to FACE $(310,415 \pm 18,628$ vs. $169,218 \pm 9,755)$.

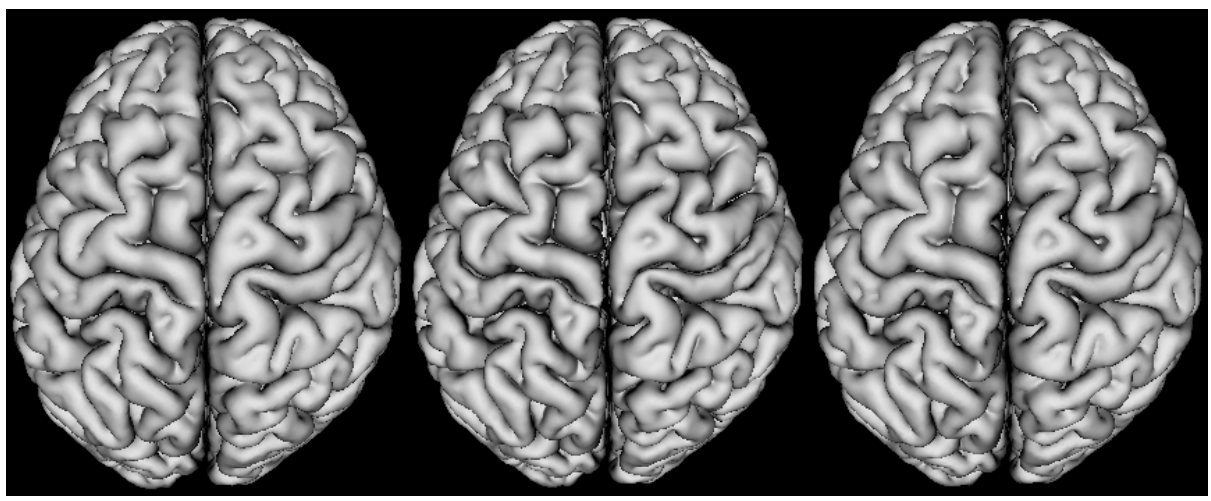

Fig. 4. Left: Surface extracted from original scan by FACE. Middle: Reconstruction from phantom by FreeSurfer. Right: Reconstruction from phantom by FACE. 
Table 1. Errors measured by the four metrics on both WM and GM surfaces. Errors are deviation from the reference surfaces. For each metric the performance on both FreeSurfer and FACE phantoms is compared for the two methods (two-tailed paired t-test). Significant smaller errors are marked by bold font.

\begin{tabular}{l|rrc|rrr} 
& \multicolumn{3}{|c|}{ FreeSurfer Phantom } & \multicolumn{3}{c}{ FACE Phantom } \\
\hline Metric & FreeSurfer & FACE & P-value & FreeSurfer & FACE & P-value \\
\hline WM $\Delta$ vol (\%) & $\mathbf{1 . 2} \pm \mathbf{1 . 1}$ & $5.4 \pm 2.6$ & 0.00 & $\mathbf{1 . 7} \pm \mathbf{1 . 9}$ & $4.9 \pm 2.3$ & 0.00 \\
WM $\Delta$ area (\%) & $7.6 \pm 1.9$ & $\mathbf{3 . 1} \pm \mathbf{1 . 5}$ & 0.00 & $15.4 \pm 3.4$ & $\mathbf{9 . 4} \pm \mathbf{1 . 9}$ & 0.00 \\
Brain $\Delta$ vol (\%) & $4.4 \pm 1.2$ & $4.0 \pm 1.0$ & 0.36 & $5.5 \pm 2.0$ & $\mathbf{3 . 7} \pm \mathbf{0 . 8}$ & 0.01 \\
GM $\Delta$ area (\%) & $5.4 \pm 1.4$ & $5.0 \pm 3.1$ & 0.54 & $2.5 \pm 2.4$ & $1.6 \pm 1.5$ & 0.22 \\
WM FN (\%) & $8.5 \pm 1.3$ & $\mathbf{4 . 2} \pm \mathbf{0 . 6}$ & 0.00 & $10.0 \pm 2.1$ & $\mathbf{3 . 2} \pm \mathbf{0 . 6}$ & 0.00 \\
WM FP (\%) & $\mathbf{7 . 8} \pm \mathbf{0 . 8}$ & $9.1 \pm 2.3$ & 0.01 & $8.7 \pm 0.8$ & $\mathbf{7 . 4} \pm \mathbf{1 . 8}$ & 0.00 \\
GM FN (\%) & $23.4 \pm 1.3$ & $\mathbf{2 1 . 9} \pm \mathbf{2 . 0}$ & 0.01 & $26.4 \pm 3.7$ & $\mathbf{1 9 . 9} \pm \mathbf{1 . 9}$ & 0.00 \\
GM FP (\%) & $15.7 \pm 1.4$ & $\mathbf{7 . 3} \pm \mathbf{1 . 4}$ & 0.00 & $17.6 \pm 3.1$ & $\mathbf{6 . 9} \pm \mathbf{1 . 3}$ & 0.00 \\
WM ref2test (mm) & $0.95 \pm 0.64$ & $1.14 \pm 0.11$ & 0.20 & $1.47 \pm 0.90$ & $\mathbf{0 . 6 3} \pm \mathbf{0 . 0 7}$ & 0.00 \\
WM test2ref (mm) & $0.75 \pm 0.14$ & $0.84 \pm 0.17$ & 0.13 & $1.28 \pm 0.17$ & $\mathbf{0 . 4 6} \pm \mathbf{0 . 0 5}$ & 0.00 \\
GM ref2test (mm) & $0.86 \pm 0.50$ & $1.07 \pm 0.11$ & 0.08 & $1.26 \pm 0.92$ & $\mathbf{0 . 6 4} \pm \mathbf{0 . 0 8}$ & 0.02 \\
GM test2ref (mm) & $0.83 \pm 0.14$ & $\mathbf{0 . 6 3} \pm \mathbf{0 . 1 3}$ & 0.00 & $1.39 \pm 0.19$ & $\mathbf{0 . 5 9} \pm \mathbf{0 . 0 6}$ & 0.00 \\
\hline
\end{tabular}

Table 1 lists the results for each error metric averaged for the 16 subjects. The errors of the two methods for each metric was compared and tested by two-tailed paired t-test (the p-values are listed in the right hand column of each phantom). Significant smaller errors are marked by bold font. The volume and area errors are absolute percent change compared the to reference surfaces. The under/over segmentation error is measured by percent outside reference surface volume (false positives (FP)) and percent missing inside reference surface (false negatives $(\mathrm{FN})$ ). The explicit geometrical difference is measured by the RMS error in $\mathrm{mm}$.

\section{Discussion}

From table 1 it can be observed that FACE has significantly fewer WM false negatives and GM false positives when testing on both groups of phantoms. The two metrics are related in that missing WM voxels most likely are classified as GM voxels. Generally, both methods seem to over-expand the surfaces when compared to the phantoms. This especially increases the GM false negatives percentage, as the GM tissue class is smaller than the WM tissue class.

The geometrical error rates show that the average distance between the test and reference surfaces is at subvoxel level when testing the accuracy of FACE. Reproducibility errors of FACE are consistently around half a voxel size, while FreeSurfer reproducibility errors are between 0.75 - 0.95 voxel size. For purposes of comparison the difference for the reference surfaces of the two methods was measured to $1.48 \pm 0.31 \mathrm{~mm}$ (average for both WM and GM surfaces).

When looking at the volume and area errors for the GM surfaces, i.e. cerebrum volume and area, there is little difference between the two methods, and the error 
is fairly small $(1.6 \%-5.5 \%)$. Also, the WM volume errors are low. However, higher error rates are found in the WM area. Looking at the area change per subject, it was found that all reconstructed WM surfaces had a smaller area than the reference, while the volume remained more or less the same. This could point to the fact that the WM voxels in the phantoms do not exactly resemble the original MR WM voxels leading to less deep sulci. Improvements of the phantoms could solve this bias. Also, visual inspection of the surfaces revealed significant differences in the surfaces at the base of the brain due to the different brain stem cutting strategies in the two methods. The inspection also revealed that FreeSurfer in a few surfaces missed part of the occipital lobe. This could be caused by registration errors which again could be caused by tissue voxels not resembling real MR data.

Generally, the tests show that the accuracy of FACE is comparable to FreeSurfer. In most cases FACE has a significantly better accuracy. FACE is on average more than 25 times faster than FreeSurfer. The longer extraction time in FreeSurfer can partly be explained by the high number of vertices in the surfaces. FreeSurfer generates surfaces with almost twice the number of vertices compared to FACE. Another reason for the speed difference is a very fast convergence of the deformation in FACE due to refraining from minimising an objective function.

Even though FACE in the comparison proved to be more accurate, results from some of the error metrics and visual inspections suggested that the phantoms could be improved to resemble real anatomical MR data. However, the results indicate that FACE is comparable to FreeSurfer in terms of accuracy.

The subjects used in this study were healthy without altered cortical morphology. Further studies must examine the accuracy of the two methods when analysing subjects with altered morphology (e.g. Alzheimer's patients), which is often the case in clinical trials.

\section{Acknowledgements}

Test data were provided courtesy of Dr. Peter Johannsen, Rigshospitalet, under grant number 22-04-0458 Danish Medical Research Counsil, and the International Consortium of Brain Mapping, McConnell Brain Imaging Centre, Montreal Neurological Institute, McGill University.

\section{References}

1. Cohen, L.D., Cohen, I.: Finite-element methods for active contour models and balloons for 2D and 3D images. IEEE Trans. Pattern Analysis and Machine Intelligence (1993)

2. Mangin, J.F., Frouin, V., Bloch, I., Régis, J., López-Krahe, J.: From 3d magnetic resonance images to structural representations of the cortex topography using topology preserving deformations. Journal of Mathematical Imaging and Vision 5(4), 297-318 (1995)

3. McInerney, T., Terzopoulos, D.: Topology adaptive deformable surfaces for medical image volume segmentation. IEEE Trans. Medical Imaging 18(10), 840-850 (1999) 
4. Dale, A.M., Fischl, B., Sereno, M.I.: Cortical surface-based analysis i: Segmentation and surface reconstruction. NeuroImage 9(2), 179-194 (1999)

5. Zeng, X., Staib, L.H., Schultz, R.T., Duncan, J.S.: Segmentation and measurement of the cortex from 3-d mr images using coupled-surfaces propagation. IEEE Trans. Medical Imaging 18(10), 100-111 (1999)

6. Han, X., Pham, D., Tosun, D., Rettmann, M., Xu, C., Prince, J.: Cruise: Cortical reconstruction using implicit surface evolution. NeuroImage 23(3), 997-1012 (2004)

7. Kim, J.S., Singh, V., Lee, J.K., Lerch, J., Ad-Dab'bagh, Y., MacDonald, D., Lee, J.M., Kim, S.I., Evans, A.C.: Automated 3-d extraction and evaluation of the inner and outer cortical surfaces using a laplacian map and partial volume effect classification. NeuroImage 27(1), 210-221 (2005)

8. Eskildsen, S.F., Uldahl, M., Østergaard, L.R.: Extraction of the cerebral cortical boundaries from mri for measurement of cortical thickness. Progress in Biomedical Optics and Imaging - Proceedings of SPIE 5747(2), 1400-1410 (2005)

9. Eskildsen, S.F., Østergaard, L.R.: Active surface approach for extraction of the human cerebral cortex from mri. In: Larsen, R., Nielsen, M., Sporring, J. (eds.) MICCAI 2006. LNCS, vol. 4191, Springer, Heidelberg (2006)

10. Lee, J., Lee, J.M., Kim, J.S., Kim, I.Y., Evans, A.C., Kim, S.I.: A novel quantitative cross-validation of different cortical surface reconstruction algorithms using mri phantom. NeuroImage 31, 572-584 (2006)

11. Fischl, B., Sereno, M.I., Dale, A.M.: Cortical surface-based analysis ii: Inflation, flattening, and surface-based coordinate system. NeuroImage 9(2), 195-207 (1999)

12. Collins, D.L., Neelin, P., Peters, T.M., Evans, A.: Automatic 3d intersubject registration of $\mathrm{mr}$ volumetric data in standardized talairach space. Journal of Computer Assisted Tomography 18(2), 192-205 (1994)

13. Smith, S.M.: Fast robust automated brain extraction. Human Brain Mapping 17(3), 143-155 (2002)

14. Chen, L., Wagenknecht, G.: Automated topology correction for human brain segmentation. In: Larsen, R., Nielsen, M., Sporring, J. (eds.) MICCAI 2006. LNCS, vol. 4191, pp. 316-323. Springer, Heidelberg (2006)

15. Kwan, R.S., Evans, A., Pike, G.: MRI simulation-based evaluation of imageprocessing and classification methods. IEEE Transactions on Medical Imaging 18(11), 1085-1097 (1999) 